\title{
Incidence of symptomatic Venous Thrombo Embolism (VTE), with or without chemoprophylaxis, in patients undergoing Total Knee Replacement: A comparative study
}

\author{
Authors \\ Tom Jose ${ }^{1}$, Murukan Babu², Georgy J Kuruvilla ${ }^{3}$ \\ ${ }^{1}$ Consultant Orthopaedic Surgeon, Department of Orthopedics, Rajagiri Hospital, Chunagamvely, Aluva, \\ Ernakulam District, Kerala India \\ E mail: tomjoseblore@gmail.com \\ ${ }^{2}$ Head of the Department, Department of Orthopedics, Rajagiri Hospital, Chunagamvely, Aluva, Ernakulam \\ District, Kerala, India \\ Email: babumurukan@gmail.com \\ ${ }^{3}$ Consultant Orthopaedic Surgeon, HGM Hospital Muttuchira, Kottayam, Kerala, India \\ Email: georgy.kuruvilla@gmail.com
}

\begin{abstract}
Background: The incidence of Venous Thrombo Embolism (VTE) in Western population undergoing major orthopaedic surgery without any thromboprophylaxis has been reported to range from $32 \%$ to $88 \%$. But there is a firm belief that this complication is rare in Asian patients. Majority of publications on thromboembolic disease are from the West and some studies from Asia often contradict this. They say, routine chemoprophylaxis is perhaps not justified in every patient, instead high level of suspicion and close clinical monitoring is only what is required. The issue has acquired greater significance in Indian subjects in recent times as there is an exponential increase in the number of joint replacement surgeries and surgeries for lower limb fractures. Very few studies have been published from India on the subject and very little is known about the true incidence of the condition. In this context this study was designed.

Objectives: To study the incidence of symptomatic VTE in patients with and without chemoprophylaxis Methods: 110 patients who underwent Total Knee Replacement (26 bilateral and 84 unilateral knees) during a period between October 2011 and October 2013 were studied for the occurrence of symptomatic VTE. All of these,110 patients received chemoprophylaxis. Each of these patients were assessed for 1 month for any clinical signs or symptoms that may show the presence of underlying VTE-such as, fever, calf swelling, pain or tenderness of calf. If there was suspicion of VTE, an ultrasound examination was carried out and VTE was confirmed. The results from this study were compared with 57 patients who underwent surgery during the period 2008 -2011 who received no chemoprophylaxis.

Results: Among the 110 patients who received chemoprophylaxis only one person developed symptomatic VTE (0.9\%). There was no significant bleeding or wound healing problems which needed specific procedures or discontinuation of treatment. In the group of 57 which didn't receive chemoprophylaxis, 5 patients developed symptomatic VTE (8.7\%).

Conclusion: The study shows a significant reduction in VTE with chemoprophylaxis and hence routine chemoprophylaxis in every patient undergoing Total Knee Replacement is clearly justified

Keywords-Venous thromboembolism, Chemoprophylaxis, Total Knee Replacement, Deep vein thrombosis.
\end{abstract}




\section{INTRODUCTION}

Venous thromboembolism (VTE), which consists of deep vein thrombosis (DVT) and pulmonary embolism (PE), is a potentially fatal disease. The incidence of Venous Thrombo Embolism (VTE) in patients undergoing major orthopaedic surgery without any thromboprophylaxis has been reported to range from $32 \%$ to $88 \%$. The majority of the studies have been conducted and published from the western countries where DVT is more commonly seen. On the other hand, very few papers have been published from this part of the world. We, therefore, have to follow the western literature for the guidelines on thromboprophylaxis for patients undergoing major lower limb surgery. Though some of the recent studies published from other Asian countries have shown that DVT is not a rarity in Asian patients as was thought earlier $(1,2,3,4)$, many say, routine chemoprophylaxis is perhaps not justified in every patient, instead high level of suspicion and close clinical monitoring is only what is required. The issue has acquired greater significance in Indian subjects in recent times as there is an exponential increase in the number of joint replacement surgeries and surgeries for lower limb fractures. Also, there are medico-legal implications of not subjecting patients undergoing lower limb surgery to some kind of thromboprophylaxis, as some people consider this an act of negligence. The need for routine pharmacological thromboprophylaxis after TJR has been questioned $(5,6,7)$ because post-operative $\mathrm{PE}$ is rare and anticoagulants increase the risk of bleeding, with its associated morbidity and mortalty. The potential complications from potent anticoagulation and the increased costcould be justified if its use reduced the number of deaths due to $\mathrm{PE}$, and the allcause mortality $(8,9,10)$. Very few studies have been published from India on the subject and very little is known about the true incidence of the condition. In this context this study was designed.

\section{METHODS}

In our centre till 2011 we never used to give routine chemoprophylaxis. During that period we noticed an increase incidence of thromboembolic episodes in patients who had undergone Replacement surgeries. Hence we started giving chemoprophylaxis as a normal routine and the patients were studied for the occurrence of Venous Throboembolism in a prospective manner.

All the patients who were admitted for Total Knee Replacement were selected. Revision cases were excluded. Patients who underwent B/L TKR in two sittings were considered as two separate cases. But the patients who underwent $\mathrm{B} / \mathrm{L}$ TKR in single sitting were considered as a single case. For each patient, all demographic details, the nature and duration of the surgery, the type of implant, presence of any co-morbid illness were documented for a possible correlation with the occurrence of DVT. Any known risk factor associated with occurrence of DVT like past history of DVT, presence of varicose veins, obesity, malignancy etc. was also recorded.

All the operations were carried out under regional (spinal \pm epidural) anesthesia, under tourniquet control. All the surgeries were done by anterior midline skin incision and medial parapatellar approach. Implants used were Zimmer, Stryker, Smith \& Nephew, Inor and Indus . Suction drains were used in all cases and kept for 24 hours .A strict protocol for mobilization of the patients following surgery was observed as a part of mechanical thromboprophylaxis $(11,12)$. Chemoprophylaxis agent when given was started on the day of surgery. The molecules used were Enoxaparin alone and with aspirin ${ }^{13}$ and rivaroxaban .These were continued till the date of discharge usually 5th post op day. When aspirin was used along with other agents it was started on POD5 and was given for 1 month.

After operation, the patients were observed closely for signs of DVT or pulmonary embolism $(14,15,16)$. The clinical signs documented were Homans' sign, prominence of superficial veins, leg and ankle swelling, skin discoloration and fever for DVT and any respiratory symptoms for PE. If there was suspicion of DVT, a doppler examination was carried out and assessed for DVT. A diagnosis of 
DVT was made where there was visualization of thrombosis, absence of flow, lack of compressibility or lack of augmentation. Usually patients were discharged on the POD5 for unilateral cases and on POD6 for bilateral cases with strict instructions to report back if any suspicious symptoms arise. Patients were reviewed for suture removal on 12th day and after 1 month. Patients who didn't receive chemoprophylaxis also followed same protocol for assessment and follow up.

\section{RESULTS}

Total no of patients under study were 167 , of which 110 received chemoprophylaxis and the rest 57 didn't receive it. Among this 110, there was 26 bilateral cases (single sitting) and 84 unilateral knees . Implants used were from Zimmer in 95 patients, Stryker in 43, Smith\& Nephew in 10, Inor in 14 and Indus in 5 patients. Among the 110 patients who received chemoprophylaxis, the chemotherapeutic agent used was Enoxaparin only 35 patients, Enoxaparin with aspirin in 69 patients and Rivaroxaban in 6 patients. One person who was on rivaroxaban developed DVT. 3 patients on enoxaparin and 2 patients on Enoxaparin and aspirin developed local swelling and tenderness which needed doppler evaluation, but there was no DVT. There was haemarthrosis formation in 3 patients which needed aspiration and delayed wound healing problems were present in 2 patients. But there was no incidence of any life threatening bleeding or other complication which could be attributed to chemoprophylaxis. In the group of 57 which didn't receive chemoprophylaxis, 4 patients developed DVT and 1 patient developed PE.

\begin{tabular}{|l|c|c|c|c|c|c|c|}
\hline Group & $\begin{array}{c}\text { Number of } \\
\text { patients }\end{array}$ & $\begin{array}{c}\text { Patients } \\
\text { with } \\
\text { VTE }\end{array}$ & Percentage & $\begin{array}{c}\text { Patients } \\
\text { with } \\
\text { DVT }\end{array}$ & Percentage & $\begin{array}{c}\text { Patients } \\
\text { with PE }\end{array}$ & Percentage \\
\hline With Chemoprophylaxis & 110 & 1 & 0.91 & 1 & 0.91 & 0 & 0.00 \\
\hline Without Chemoprophylaxis & 57 & 5 & 8.77 & 4 & 7.02 & 1 & 1.75 \\
\hline
\end{tabular}

\section{DISCUSSION}

Thromboprophylaxis after Total Joint Replacement remains a topic of considerable debate among orthopaedic surgeons, $(17,18)$. In the context of public health, it is viewed as a preventable cause of morbidity and mortality. There is always an underlying conflict between the benefits of pharmacological thromboprophylaxis and the risk of clinically important postoperative haemorrhage. $(19,20)$ In our study the incidence of VTE in prophylaxis group was $0.91 \%$ and in nonprophylaxis group was $8.77 \%$ which shows the high incience of venous thromboembolism especially in the absence of prophylaxis. The patient who developed DVT in the first group was a known case of Rheumatoid Arthritis and DM. In the second group 4 patients had risk factors but one patient didn't have any. The data shows that VTE can occur in non-risk patients also. So using pharmacological prophylaxis only for the high-risk patients cannot be advocated.

\section{CONCLUSION}

The study shows a significant reduction in VTE with chemoprophylaxis when used and there was no evidence of any life threatening bleeding episodes or other complications directly related to its usage. Hence we strongly agree that routine chemoprophylaxis in every patient undergoing Total Knee Replacement is clearly justified.

\section{REFERENCES}

1. Ngoh ChinLiew, YunusGul et al. Postoperative Venous Thromboembolism in Asia: A Critical Appraisal of its IncidenceAsian Journal of Surgery Volume 26, Issue 3, July 2003, Pages 154-158

2. SC Srivastava Absence of pulmonary embolism in AsiansBr Med J, i (1964), pp. 772-773

3. KS Dhillon, A Askander, S DoraismayPosto perative deep-vein thrombosis in Asian patients is not a rarity: a prospective study of 
88 patients with no prophylaxisJ Bone Joint Surg Br, 78 (1996), pp. 427-430

4. GV Shead, R NarayananIncidence of postoperative venous thromboembolism in South IndiaBr J Surg, 67 (1980), pp. 813-814

5. Lee WS, Kim KI, Lee HJ, et al. The incidence of pulmonary embolism and deep vein thrombosis after knee arthroplasty in Asians remains low: a meta-analysis. Clin Orthop Relat Res 2013; 471:1523-1532.

6. Lee AD, Stephen E, Agarwal S, et al. Venous thrombo-embolism in India. Eur J Vasc Endovasc Surg 2009; 37:482-485.

7. Preventing venous thromboembolic disease in patients undergoing elective hip and knee arthroplasty evidence-based guideline and evidence report Adopted by the American Academy of OrthoPaedic Surgeons Board of Directors September 23, 2011

8. Chung LH, Chen WM, Chen CF, Chen TH, Liu CL. Deep vein thrombosis after total knee arthroplasty in Asian patients without prophylactic anticoagulation. Orthopedics 2011; 34:15.

9. Nikolaou, V.S., Desy, N.M., Bergeron, S.G. et al, Total knee replacement and chemical thromboprophylaxis: current evidence. Curr Vasc Pharmacol. 2011;9:33 ([Review]).

10. Kim, Y.H., Kim, J.S. Incidence and natural history of deep-vein thrombosis after total knee arthroplasty: a prospective randomized study. J Bone Joint Surg Br. 2002;84-B:566.

11. Shepherd A, Mills C Fatal pulmonary embolism following hip and knee replacement: a study of 2153 cases using routine mechanical prophylaxis and selective chemoprophylaxis. Hip Int 2006;16:53-6.

12. Lachiewicz PF, Soileau ES. Mechanical calf compression and aspirin prophylaxis for total knee arthroplasty. Clin Orthop 2007;464:61-64.
13. Geerts WH, Bergqvist D, Pineo GF, et al. Prevention of venous thromboembolism: American College of Chest Physicians evidence-based clinical practice guidelines (8th edi.). Chest 2008(Suppl);133:381-453.

14. Leclerc JR, Gent M, Hirsh J, et al. The incidence of symptomatic venous thromboembolism during and after prophylaxis with enoxaparin a multi-institutional cohort study of patients who underwent hip or knee arthroplasty. Arch Intern Med 1998;158: 873 -878 .

15. Cusick LA, Beverland DE. The incidence of fatal pulmonary embolism after primary hip and knee replacement in a consecutive series of 4253 patients. J Bone Joint Surg [Br] 2009;91-B:645-648.

16. Altintaş F, Gürbüz H, Erdemli B, et al. Venous thromboembolism prophylaxis in major orthopaedic surgery: a multicenter, prospective, observational study. Acta Orthop Traumatol Turc 2008;42:322-327

17. Dorr LD, Gendelman V, Maheshwari AV, et al. Multimodal thromboprophylaxis for total hip and knee arthroplasty based on risk assessment. J Bone Joint Surg [Am] 2007;89-A:2648-2657.

18. Lachiewicz PF, Soileau ES. Multimodal prophylaxis for THA with mechanical compression. Clin Orthop 2006;453:225-230.

19. Callaghan JJ, Warth LC, Hoballah JJ, Liu SS, Wells CW. Evaluation of deep venous thrombosis prophylaxis in low-risk patients undergoing total knee arthro- plasty. J Arthroplasty 2008;23(Suppl):20-24.

20. Sachs RA, Smith JH, Kuney M, Paxton L. Does anticoagulation do more harm than good?: a comparison of patients treated without prophylaxis and patients treated with low-dose warfarin after total knee arthroplasty. JArthroplasty 2003;18:389-395 